

\title{
Defect Chemical Nature of Solids as a Huge Resource of Advanced Electrochemical Technologies
}

\section{Junichiro MIZUSAKI}

Ionic species are mobile in almost every kind of materials at any state. Plasma as gaseous electrolyte, aqueous and nonaqueous electrolyte, molten salts, various kinds of solid including polymers, glasses and crystalline inorganic compounds, bio systems and so on...... It is a miracle in the cosmos that our earth surface is kept at nearly 1 bar at temperatures at which $\mathrm{H}_{2} \mathrm{O}$ is liquid. The liquid $\mathrm{H}_{2} \mathrm{O}$ is stable in only $\Delta 100 \mathrm{~K}$ in the unlimitedly wide temperature range in the cosmos. It happens that a large part of the earth surface is covered with aqueous electrolyte (seawater) and the organs of plants and creatures can not get life without plenty of aqueous electrolyte filled in them. Aqueous electrochemical phenomena govern our biosphere. In due course, a large part of electrochemical researches has been devoted on aqueous one. However, the aqueous electrolyte is perhaps the most complicated one among various electrolytes. Excess oxygen or hydrogen dissolves in water to cause variation of the potential of electrons. Water itself dissociates into ions at very low equilibrium constant, in which several ionic species are dissolved to customize the nature of the solution, resulting in the complicated equilibrium relationship. The interfacial phenomena such as concentration polarization and ionic double layer formation may be the typical appearance of this complexity.

The dawn of civilization is marked by the start of utilization of artificial tools. When humankind found how to prepare pottery, metallic tools, and began considering how to protect metallic tools and ornaments from gaseous corrosion, the application of "solid state electrochemistry" was actually started. Of course, its scientific backgrounds such as ionic diffusion process in solids, chemical potential gradient as the driving force of diffusion, thermodynamic stability of solid and so on had not been revealed until 1930's by C. Wagner, N. F. Mott, W. Schottky and others.

In solid electrolytes, there are neither such problem as solvent and solute nor such effect as concentration polarization in thin layers. The system is much simpler than the aqueous electrolyte. However, this simplicity often puzzles researchers because most of the textbooks on electrochemistry describe the "electrochemistry customized only for aqueous electrolyte". All the components in crystalline inorganic compounds are more or less ionized and mobile, where ionic transport takes place via defects or disorder of the crystalline lattice. Some crystal structures preserve such disorder intrinsically. In most crystals, nonstoichiometric deviation in composition or doping of aliovalent impurities induces disorders or defects. However, such deviation or doping also causes the variation in electronic carrier concentration. Thus, solid state electrochemistry is strongly linked with the studies on crystalline and electronic structure and thermodynamics of solids, so-called "defect chemistry."

Today, innovation is going on by the control of nonstoichiometry and defect concentration of solid compounds in advanced electrochemical technologies such as lithium ion batteries, solid oxide fuel cells, chemical sensors, utilization of compound semiconductors/magnetic materials and the control of catalysts. Progress is going on in the basic science of nonstoichiometry and defect chemistry of solids, with the development of novel experimental techniques, deepening theoretical insights. This special issue is planned to gather up and display the original papers from every frontier of the basic science and application of solid state electrochemistry.

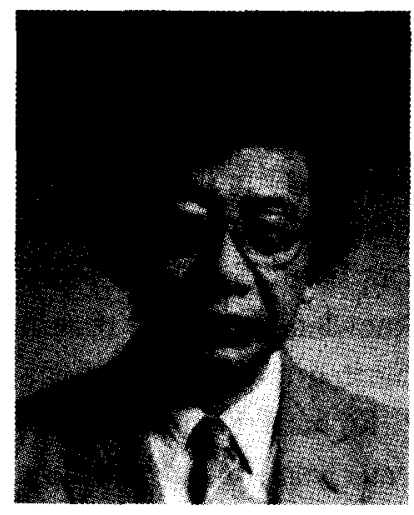

Prof. Junichiro MIZUSAKI Research Institute for Sceintific Measurements Tohoku University 